\title{
Assessing the Risk of Zika Virus to Hong Kong Residents through invasion of Infected Mosquitoes through Seaports
}

\author{
Thomas M. Kollars, Jr. \\ ${ }^{1}$ College of Health Sciences, Liberty University, USA, Former Brigade Surgeon, $415^{\text {th }}$ Chemical Brigade, \\ USAR; tkollars@liberty.edu
}

\begin{abstract}
The global expansion of Zika virus is of great concern to medical and public health officials. Identifying routes of entry into areas where suitable vectors are present and providing information concerning integrated mosquito management is critical to the prevention and control of Zika virus and its vectors. The Bioagent Transport and Environmental Modeling System (BioTEMS) has been used for modeling and planning of defense against biological weapons of mass destruction and infectious disease. Because of the important role maritime ports often play in the invasion of medically important mosquito species, port areas on the northern shore of Hong Kong Island were evaluated for susceptibility of introduction of ZIKAV. Integrated mosquito management zones and surveillance sites were identified. BioTEMS has been used to accurately predict the expansion of Zika virus in Brazil and the USA. In Hong Kong, BioTEMS accurately predicted where local dengue virus transmission occurred. BioTEMS was used to evaluate the risk of Zika virus invasion through the northern ports of Hong Kong and to provide information for vector control and surveillance. Medical personnel and public health officials should prioritize surveillance for ZIKAV in the port areas in Hong Kong. Integrated mosquito management plans should be put into place before ZIKAV arrives ashore, by either infected mosquitoes or infected humans. This information can assist in optimizing preventive medicine measures, including pre-viral invasion, serology collection, public education in personal mosquito control measures, mosquito surveillance, testing and control.
\end{abstract}

Keywords: Aedes, arbovirus, invasive species, medical countermeasures, biological agents

\section{Introduction}

Zika virus (ZIKAV) is a positive-sense single-stranded RNA virus in the family Flaviviridae. Spondweni virus is the most closely related flavivirus; other mosquito-borne viruses within this family include dengue (DENV), West Nile (WNV), yellow fever (YFV), chikungunya (CHIKV) (Kuno and Chang 2008; Lanciotti et al. 2008). ZIKAV, DENV, and CHIKV are spreading rapidly on a global scale. In addition, medically important mosquito species are capable of invading new geographic regions and exploiting niches that are similar to their natural home ranges. The principle factor responsible for the introduction of disease vectors is air and ship transport (Tatem et al. 2006). These invasive mosquito species, and the pathogens they transmit, pose significant medical and veterinary health risks in naïve populations. Although Aedes aegypti has been recorded in Hong Kong, in a study conducted during 2004, only Ae. albopictus were collected (Rueda et al. 2004). Aedes albopictus, which can transmit ZIKAV, and approximately 20 other viruses, is now one of the most common mosquito species in Hong Kong (Wong et al. 2010). Perhaps like many areas, Ae. albopictus has supplanted Ae. aegypti. This may have some effect in how rapidly an arbovirus such as Zika virus may spread in Hong Kong, as Ae. albopictus does not appear to be as an efficient a vector as Ae. aegypti. Because of the import role maritime ports often play in the invasion of medically important mosquito species, ports on the northern shore of Hong Kong Island were evaluated for susceptibility of ZIKAV, and integrated mosquito management (IMM) zones and surveillance sites were identified.

\section{Methods}

ArcGIS geospatial analysis software, Statistica statistical software and the Bioagent Transport and Environmental Modeling System (BioTEMS) were used to analyze geographic information and conduct data analysis. BioTEMS has previously been used for modeling biological weapons defense and infectious diseases in several countries (Kollars 2008) and as part of the author's duties as Epidemiologist (Consequence Management Unit) and as Brigade Surgeon $\left(415^{\text {th }}\right.$ Chemical Brigade USAR). Ecological niche and dynamic change modeling are used within BioTEMS to identify areas at risk for invasion by vectors, arboviruses and provide information for integrated mosquito management (Kollars et al. 2016). BioTEMS has been used in risk analysis and vulnerability assessment for biological weapons of mass destruction, including; Bacillus anthracis, Francisella tularensis, Yersinia pestis, and Crimean-Congo hemorrhagic fever virus for security for presidential/national events, government facilities, and populations. BioTEMS has also be used with HPAC to analyze bioagent information and to optimize placement of Biological Integrated Detection Systems. In 
Assessing The Risk Of Zika Virus To Hong Kong Residents Through Invasion Of Infected ...

addition to applications for biological weapons defense and medical countermeasures, BioTEMS has been adapted for use for infectious disease modeling and planning.

The BioTEMS TIGER model was developed to assist in identifying areas at highest risk for invasive mosquito species and pathogens and to optimize surveillance and control efforts (Kollars et. al. 2016). Within the BioTEMS TIGER model; Transport- identifies the point of origin, method and rate of transport to a locality, Introduction- point/area of initial introduction/immigrant haplotypes and preliminary spread into a locality, Gapdetermines the area where vector/pathogen initially spreads once it has gained a foothold, Escalade- incorporates abiotic and biotic resistance to invasion, and Residence and recruitment - incorporates factors and area where vector/pathogen adds to genetic diversity or becomes endemic and recruits con-specifics. The BioTEMS model has been used to identify areas at risk for ZIKAV and identify areas for integrated mosquito management in maritime ports in Miami, Tampa, and Mayport Naval Port in Florida, and the ports of Rio de Janeiro and Fortaleza, in Brazil (Kollars and Kollars 2016; Kollars 2017). The BioTEMS model was used to identify IMM zones and surveillance sites to assist in mitigashould Zika virus be introduced through the northern ports of Hong Kong. Other Hong Kong ports were not evaluated during this study. BioTEMS and ArcView were used to produce output into Google Earth.

\section{Results And Discussion}

Hong Kong Island is at risk for the introduction and spread of ZIKAV during the initial invasion of the virus through mosquitoes or humans in the port areas infecting local Ae. albopictus or by introduction of infected haplotypes (Figure 1). The BioTEMS model was validated and accurately predicted the presence of local transmission of dengue virus at the Zoological Garden from a published report (Poon et al. 2015). Approximately $15 \mathrm{~km}^{2}$ surrounding the northern port areas are at high risk for the Introduction/Invasion of ZIKAV infected Aedes mosquitoes. An additional $10 \mathrm{~km}^{2}$ of IMM zone should be added to the control measures and surveillance conducted at the Introduction/Invasion zone to reduce the risk of Gap Infiltration by infected mosquitoes. Surveillance sites around the ports as well as other areas were identified to assist in surveillance. The BioTEMS model has been used to identify areas at risk for ZIKAV and identify areas for integrated mosquito management in other ports, e.g. Miami (Florida), United Arab Emirates, Hong Kong, Malaysia, Republic of Georgia, Honduras, Australia and Brazil. Import of ZIKAV into Hong Kong is of high probability if the port of origin is endemic or of infected mosquitoes or personnel arrive through transit. BioTEMS identified areas where ZIKAV could spread if introduced into the port. Pre-invasion serology and samples should be preserved should ZIKAV be introduced into Hong Kong. This epidemiologic information would provide valuable insight for medical and public health authorities in controlling the spread of the infection in Hong Kong and to assist other port cities in developing medical countermeasure to ZIKAV. Clinical evaluation with laboratory testing are essential for the diagnosis of Zika virus infection because of clinical overlap with other arboviruses, such as DENV and CHIKV; with molecular diagnostics during the acute phase being the most specific diagnostic approach (Plourde and Block 2016).

Environmentally friendly mosquito control measures are essential, particularly in urban environments. Recently a new pesticide, Entobac, was tested which has both adulticidal and larvacidal activity. The pesticide is non-toxic and has been tested in port areas and when used in applicators such as the ProVector Tube, the pesticide remains active up to 3 months (Kollars et al. 2016). The technology used in the ProVector allows for the adult mosquito to ingest the pesticide and then transport the pesticide to larval breeding sites. In approximately 7 days the adult mosquito dies and if the mosquito lands in water, the larval mosquitoes die as well. The ProVector may provide a useful tool in killing larva mosquitoes in various breeding sites around ports, such as in waste tires. The ProVector tube can be hung from structures in the port areas and on ships to help reduce the risk of ZIKAV infected mosquitoes from being imported into Hong Kong. In the event an infected traveler infects local mosquitoes, the presence of ProVector applicators, or other long lasting environmentally safe pesticides, can reduce the mosquito population and reduce the risk of ZIKAV spreading. The information provided by BioTEMS can assist in optimizing preventive medicine measures in specific areas in Hong Kong, including pre-viral invasion serology collection, public education concerning personal mosquito control measures, mosquito surveillance, testing and control.

\section{Conclusion}

This study shows that Hong Kong may be vulnerable to Zika virus and other arboviruses that are transmitted by Ae. albopictus, as shown by the previous invasion of dengue virus into the mosquito population (Poon et al. 2015). Identifying the correct pesticide to avoid resistance or environmental contamination is an important aspect of IMM. Focusing control efforts primarily on travelers and not including ports of entry may do a disservice to the population to whom public health officials are trying to protect. Public health policy should include epidemiologic surveillance in the residents and visitors to Hong Kong in and around sea and air ports and integrated mosquito management plans should be put into place before ZIKAV arrives ashore. The 
Assessing The Risk Of Zika Virus To Hong Kong Residents Through Invasion Of Infected ...

BioTEMS model may also be useful in identifying additional marine and air ports in China that are at risk from invasive mosquito species and the arboviruses they transmit.

\section{Acknowledgment}

The views expressed in this publication are those of the author and do not reflect the official policy of the United States Army or United States Government.

\section{References}

[1]. Lanciotti RS, Kosoy OL, Laven JJ, Velez JO, Lambert AJ, Johnson AJ, et al. Genetic and serologic properties of Zika virus associated with an epidemic, Yap State, Micronesia, 2007. Emerg Infect Dis. 2008; 14:1232-9.

[2]. Kollars, TM (2008) BioTEMS-Biology based modeling to determine Bioagent fate. Chemical Biological Weapons Delivery Methods and Consequence Assessment Modeling Conference. National Geospatial Intelligence Center: Weapons Intelligence, Nonproliferation and Arms Control.

[3]. Kollars, TM Jr, Kollars PG, and B Hulsey B (2016). Reducing the Risk to Marine Ports from Invasive Mosquito Species, Zika, Dengue, Chikungunya viruses and Filariasis. Intl J Med. 4:70-73.

[4]. Kollars, TM and Kollars, JW. (2016) The invasion of Zika virus into Rio de Janeiro and Fortaleza, Brazil, inside out or outside in? Ann Community Med and Pract. 2: 1-4.

[5]. Kollars, TM Jr. (2017). Assessing likely invasion sites of Zika virus-infected mosquitoes in civilian and naval maritime ports in Florida. Res Rep Trop Med 8: 1-6.

[6]. Kuno G, Chang GJ. Full-length sequencing and genomic characterization of Bagaza, Kedougou, and Zika viruses. Arch Virol. 2007;152:687-96.

[7]. Plourde, Anna R., and Evan M. Bloch. "A literature review of Zika virus." Emerging Infectious Diseases, vol. 22, no. 7, 2016, p. 1185-1192.

[8]. Poon CM and Lee SS (2015). Has dengue found its home in Hong Kong? Hong Kong Med J. 21:85-7

[9]. Rueda LM, Pecor JM, Yuen, MC, Lee MW (2004). New record, habitats, and updated checklist of the mosquitoes of Hong Kong. J. Mosq. Control 20: 204-207.

[10]. Tatem AJ, SI Hay SI, Rogers DJ (2006). Global traffic and disease vectors. Proc Natl Acad Sci USA. $103: 6242-6247$.

[11]. Wong MC, Mok HY, Ma HM, Leeb MW and Fokb MY (2011). A climate model for predicting the abundance of Aedes mosquitoes in Hong Kong. Meteorol. Appl. 18: 105-110.

Figure 1. Northern port areas of Hong Kong at high risk for the introduction of Zika virus (red) and recommended integrated mosquito management (yellow) should Zika virus be introduced through the sea ports. Recommended surveillance sites for mosquito surveillance (blue circles). Dengue hot zone where local transmission likely occurred (green diamond).

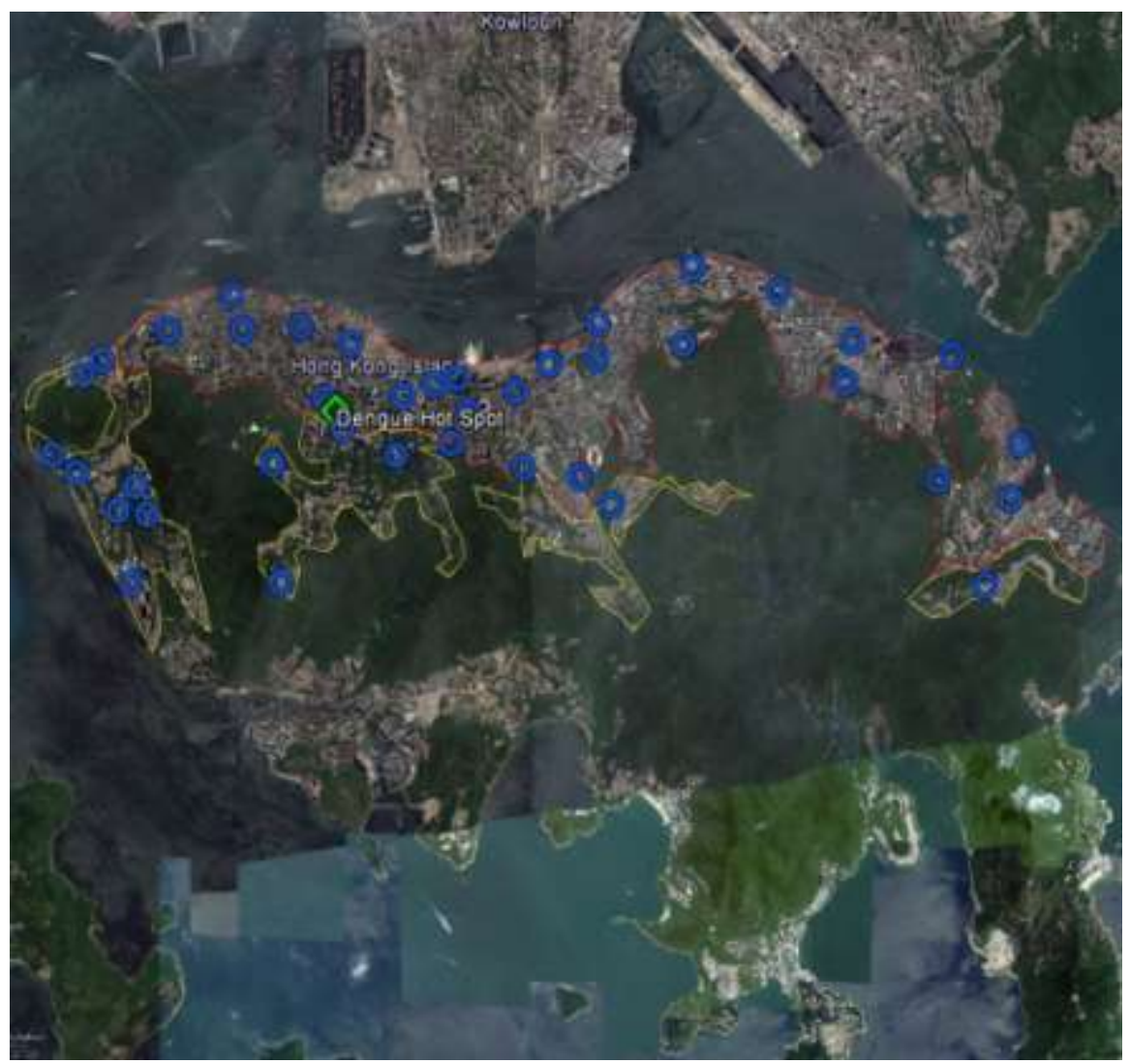

